\title{
PKH26 Can Transfer to Host Cells In Vitro and Vivo
}

\author{
Peng Li, ${ }^{1-3}$ Run Zhang, ${ }^{1-3}$ Haitao Sun, ${ }^{1-3}$ Lei Chen, ${ }^{1-3}$ Fang Liu, ${ }^{4}$ Chen Yao, \\ Mouxuan Du, ${ }^{1-3}$ and Xiaodan Jiang ${ }^{1-3}$
}

The fluorescent dye, PKH26, which mainly binds to the cell membrane, has been used as the cell tracer to locate the transplanted cells in host for a long time. However, there was no detailed report that whether the PKH26 dye was specific to the transplanted cells. Therefore, the aim of this article is to explore the effect of cells debris as the cracking cells from the PKH26-labeled adipose-derived stem cells (ADSCs) on the cells in vitro and the host in vivo. After we tested the proliferation and toxicity of PKH26 to the ADSCs by the Cell Count- 8 kit and alamar blue assay, we constructed 2 models, coculturing lots of PKH26-labeled cell debris with the unlabeled ADSCs in vitro and injecting via the tail vein in rat, to evaluate the specificity of the PKH26 dye. The result indicated that the PKH26 didn't inhibit the proliferation and had no toxicity to the ADSCs compared with the unlabeled ADSCs, but the cell debris cracking from PKH26-labeled transplanted cells can cause the unlabeled cells to emit red fluorescence in vitro and also lead the tissues displaying red fluorescence in vivo. We can conclude that the PKH26 dye, used as a cell tracer for a long time, was not an ideal cell tracer.

\section{Introduction}

$\mathrm{T}$ He study of Cellular labeling in vitro and tracking in vivo is very important to understand the diseases and mechanism of therapy. $\mathrm{PKH} 3$, the initial product of fluorescent lipophilic molecular, was reported first by Slezak and Horan as early as 1989 [1]. Soon after, PKH2 [2], PKH26 [3], and PKH67 [4] were used as cell tracers in various scientific fields one by one as they shared the same characteristics as PKH3.

An ideal cell tracer should have the following necessary features: (I) have sufficiently high signal to detect, (II) not be eluted, (III) not be metabolized, and (IV) have specific to labeled cells. Shao-Xin Zheng reported that, when the PKH26-labeled cardiac stem cells and mesenchymal stem cells were traced by the confocal laser scanning microscope, the red fluorescence was found to display on muscle and endothelium [5]. S.S. Seyed Jafari confirmed that PKH26treated neural stem cells migrated to the subjacent brain parenchyma and expressed neuronal and astrocytic cell markers [6]. Because so many researchers used the PKH26 dye to label cells [7-8], it cannot be ignored whether the labeling cells transplanted into the host were specific to the host cells, especially when only a few cells survived [9-10].

Here in this article (Fig. 1), after comparing the proliferation and toxicity of PKH26 to the adipose-derived stem cells
(ADSCs) between the labeled and unlabeled cells by the Cell Count-8 kit (CCK-8), as well as alamar blue assay, we constructed 2 models to evaluate the specificity of the PKH26 dye, one was to coculture lots of PKH26-labeled cell debris (dead cells, which were used to simulate the transfer process with the treatment of hypotonicity and trypsin) with the unlabeled ADSCs in vitro; the other was to inject the rat with PKH26-labeled cell debris via the tail vein.

\section{Materials and Methods \\ ADSCs culture and label with PKH26}

All animal protocols used have been approved by the Animal Care and Use Committee of the Southern Medical University and all the procedures were carried out in accordance with institutional guidelines. The adipose tissue was obtained from the subcutaneous tissue of a neonate Wistar inbred rat (5day-old). It was digested with collagenase I (Sigma Aldrich) at $37^{\circ} \mathrm{C}$ for $30 \mathrm{~min}$ and neutralized by the addition of a complete cell culture medium (Gibco). Then, the mixture was filtered and the filtrate was centrifuged. The cells were collected and cultured in the complete cell culture medium. After 5 days, when the cells reached their optimal confluency, they were passaged. ADSCs at passage (p) 2 were labeled with the red fluorescent

\footnotetext{
${ }^{1}$ Department of Neurosurgery, Southern Medical University, Zhujiang Hospital, Guangzhou, China.

${ }^{2}$ National Key Clinic Department, Key Laboratory on Brain Function Repair and Regeneration of Guangdong, Southern Medical University, Guangzhou, China.

${ }^{3}$ Neurosurgery Institute, Key Laboratory on Brain Function Repair and Regeneration of Guangdong, Southern Medical University, Guangzhou, China.

${ }^{4}$ Department of Obstetrics and Gynecology, Shihezi University School of Medicine, Shihezi, Xinjiang, China.

${ }^{5}$ Neurosurgery Department of the Affiliated Yuebei People's Hospital, Shantou University Medical College, Shaoguan, Guangdong, China.
} 


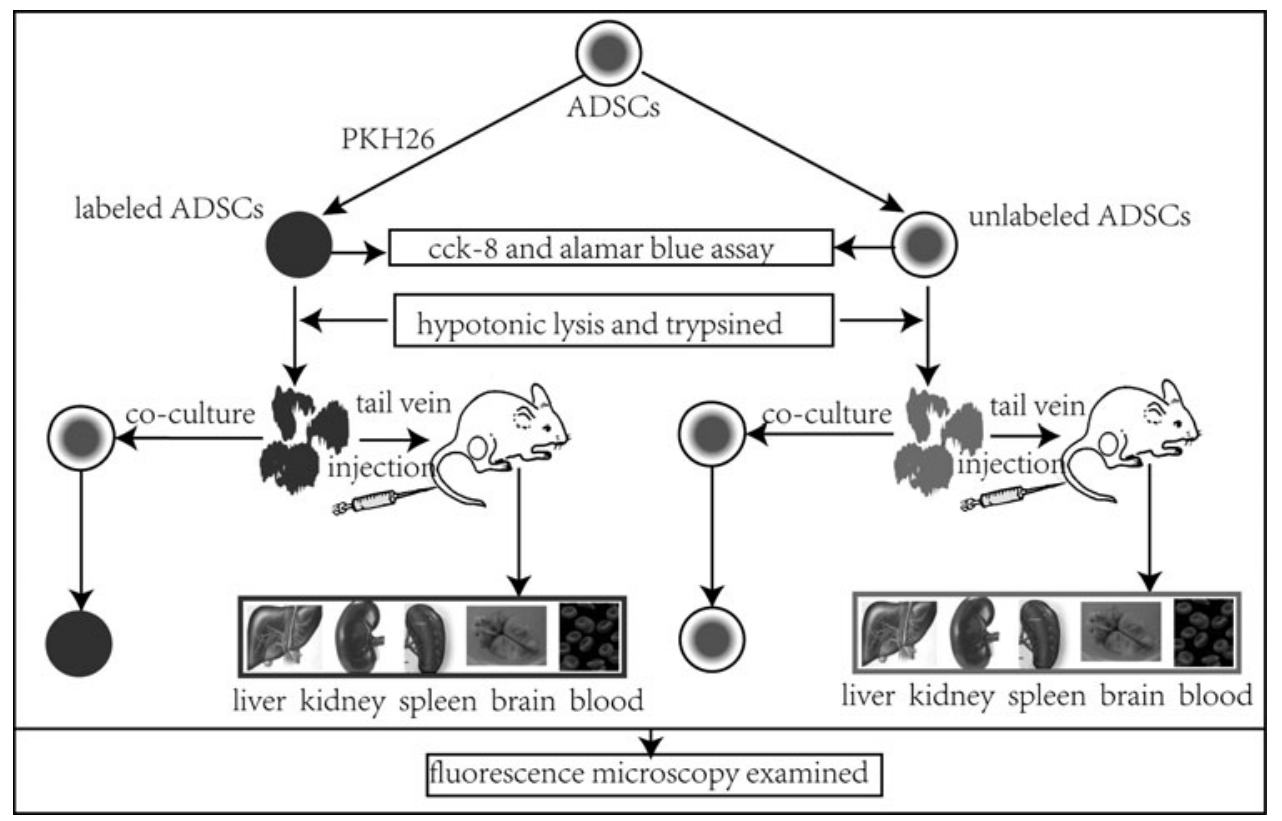

FIG. 1. Experimental design routes and steps.

dye PKH26 (sigma Aldrich) according to the manufacturer's protocol. Briefly, the detached ADSCs were washed by a serum-free medium and resuspended in $1 \mathrm{~mL}$ of dilution buffer from the manufacturer's labeling kit. The cells suspension was mixed with an equal volume of the labeling solution containing $4 \times 10^{-6} \mathrm{M}$ PKH26 in the dilution buffer and incubated for $5 \mathrm{~min}$ at room temperature (RT). After the reaction was ended by adding $2 \mathrm{~mL}$ fetal bovine serum (FBS), cells were washed 3 times with the Dulbecco's modified Eagle's medium (DMEM)/ F12 and observed by fluorescent microscopy.

\section{Analysis of proliferation with cck-8 kit}

The relative toxicity of PKH26 to the ADSCs was assessed by CCK-8 (Dojindo, Japan) as following according to the manufacturer's protocol. The groups included the PKH26-labeled group, the unlabeled group, and the blank control. First, the ADSCs were seeded in 96-well plates at 1,000 cells in $100 \mu \mathrm{L}$ DMEM/F12 and 10\% FBS per well. $100 \mu \mathrm{L}$ DMEM/F12 and $10 \%$ FBS were added into the blank control per well, and then $10 \mu \mathrm{L} \mathrm{CCK}-8$ was added in each well and incubated at $37^{\circ} \mathrm{C} 95 \%$ relative humidity, and $5 \% \mathrm{CO}_{2}$ for $2 \mathrm{~h}$ per day for 5 days. Last, the plates were measured at $450 \mathrm{~nm}$ on the spectrophotometer.

\section{Toxicity test with alamar blue}

Alamar blue [11] was used to evaluate the toxicity of PKH26 to ADSCs as following. After the ADSCs were seeded in 96well plates at 1,000 cells in $100 \mu \mathrm{L}$ DMEM/F12 and 10\% FBS per well, $10 \mu \mathrm{L}$ alamar blue was added in each well and incubated at $37^{\circ} \mathrm{C} 95 \%$ relative humidity, and $5 \% \mathrm{CO}_{2}$ for another $2 \mathrm{~h}$ for 5 days. The plates were measured at 570 (measurement) and 630 (reference) $\mathrm{nm}$ on the spectrophotometer.

\section{Hypotonic lysis of labeled ADSCs and identification}

The total number of PKH26-labeled and -unlabeled ADSCs was $5 \times 10^{5}$, respectively. After being transferred in two 15-mL tubes, the cells were placed in tubes by adding sterile water to damage the cells into debris overnight in $4^{\circ} \mathrm{C}$.
Then, the cell debris were collected by high-speed centrifuging (5000 $\mathrm{g}, 15 \mathrm{~min})$, trypsined for $1 \mathrm{~h}$ at RT, and centrifuged at the same speed after being washed with the phosphate buffer solution (PBS) for 3 times. Finally, the debris was indentified under an inverted phase-contrast microscope and fluorescent microscopy.

\section{Coculture the labeled cell debris with unlabeled ADSCs}

The cell debris from PKH26-labeled and -unlabeled ADSCs (as control) were added into unlabeled ADSCs at p2 in DMEM/F12 and 10\% FBS cultured for 7 days. The cells were washed 5 times with PBS before they were observed through fluorescent microscopy.

\section{Transplantation of PKH26-labeled cell debris via tail vein}

The cell debris from $5 \times 10^{5}$ PKH26-labeled and -unlabeled ADSCs were injected into Wistar rats ( $n=3$ /group) via the tail vein. After 7 days, the livers, kidneys, spleens, and brains were collected and examined by frozen sections, and peripheral blood was tested by smear under fluorescent microscopy. During which, 4',6-diamidino-2-phenylindole was used to stain the nuclei.

\section{Statistical analysis}

The statistical analysis was carried out with an independent sample $t$ test for the comparison of means between two groups. A 95\% confidence level and a corresponding $P$-value $<0.05$ were considered significant.

\section{Result}

\section{Morphology of ADSCS}

After the primary ADSCs were harvested, the adherent monolayer cells at P2 grew better and displayed spine-like 
morphology, the cells were also flat and had branch-like processes from the cell body under light microscopy (Fig. $2 \mathrm{~A}, \mathrm{~B})$.

\section{PKH26 did not suppress cell proliferation}

To investigate the effect of PKH26 on the proliferation and toxicity of ADSCs, the unlabeled ADSCs were used as control. As showed in Fig. 2C and D, the PKH26 could not inhibit the proliferation and had no toxicity to the ADSCs, because there was no significant difference between PKH26-labeled and -unlabeled groups by the CCK-8 kit $(P 1=0.961>0.05)$ and alamar blue assay $(P 2=0.265>0.05)$.

\section{Hypotonic lysis of the PKH26-labeled and -unlabeled ADSCs and identification}

The PKH26-labeled ADSCs were in a circular form (Fig. $3 \mathrm{~A})$ after being labeled and seeded in the cell culture plate, and displayed red fluorescence and the edge of the cells were with smooth edge (Fig. 3B) under fluorescent microscopy. The cells debris from PKH26-labeled and -unlabeled ADSCs were broken in sterile water and trypsin. There were not any complete cells under light microscopy (Fig. 3C) and the red fluorescence was displayed irregular form under fluorescent microscopy (Fig. 3D).

\section{The cell debris from PKH26-labeled ADSCs caused the unlabeled ADSCs to show red fluorescence}

To explore the effect of the dead cells on the live cells in vitro, the cells debris from PKH26-labeled and -unlabeled ADSCs were cocultured with unlabeled ADSCs for 7 days. The result indicated that unlabeled ADSCs showed red fluorescence significantly (Fig. 3F). However, ADSCs cocultured with the debris from unlabeled cells did not show red fluorescence at all (Fig. 3E). The cell debris from PKH26-labeled ADSCs led the tissues to display red fluorescence.

Cell debris from PKH26-labeled ADSCs were transplanted via rat tail vein. After 7 days, the livers, spleens, kidneys, brains, and blood smear were detected by immunofluorence microscopy. (Fig. 4A), spleens (Fig. 4C), kidneys (Fig. 4E), and blood (Fig. 4I), and the weak red fluorescence displayed in brains (Fig. 4G). However, the tissues from the control group did not find any red fluorescence beside the blue nuclei staining (Fig. 4B, D, F, J).

\section{Discussion}

In our study, the results tested by the CCK- 8 kit and alamar blue assay showed that PKH26 neither inhibited cell proliferation nor had the toxicity to the ADSCs compared with unlabeled ADSCs, respectively, as Fig. $2 \mathrm{C}$ and D, and the ADSCs labeled with the PKH26 dye showed a strong red fluorescence signal under the fluorescent microscope, which was demonstrated as Fig. 3B. As it is known to all, the hypotonic sterile water can burst the cells and the trypsin can also damage the membrane at a certain time, so the cells debris from PKH26-labeled ADSCs were obtained applying the above principle. Although the PKH26 had so many advantages as a cell-tracer biomaterial, it was unexpected that the cells debris from PKH26-labeled ADSCs could make the
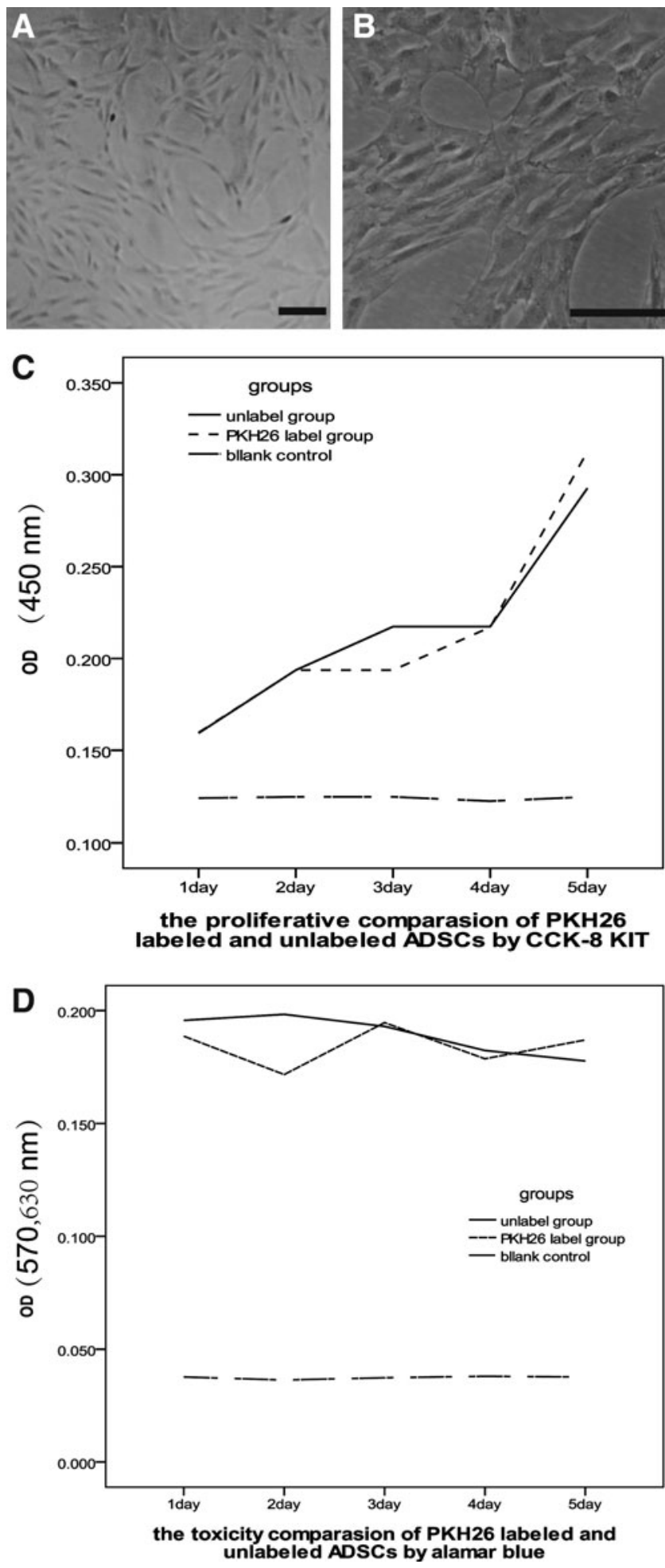

FIG. 2. The adipose-derived stem cells (ADSCs) (A, B) observed under light microscopy and the proliferative comparison by Cell Count-8 kit (CCK-8) KIT (C) and toxic comparison by alamar blue (D) after the ADSCs were labeled by PKH26 compared with unlabeled ADSCs. Bar $=100 \mu \mathrm{m}$. 

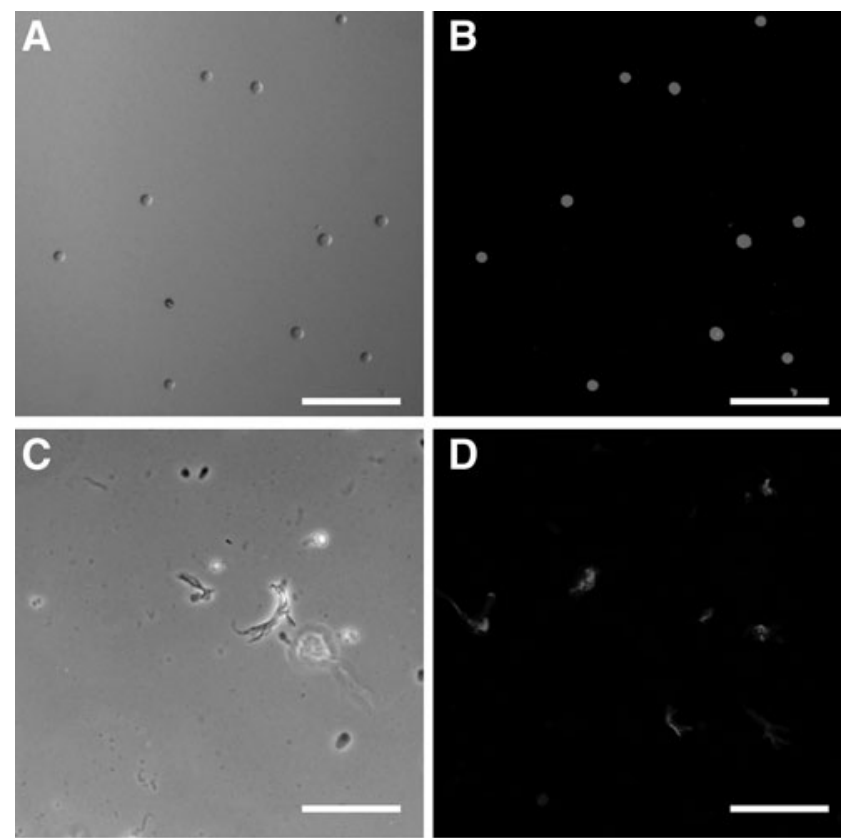

E
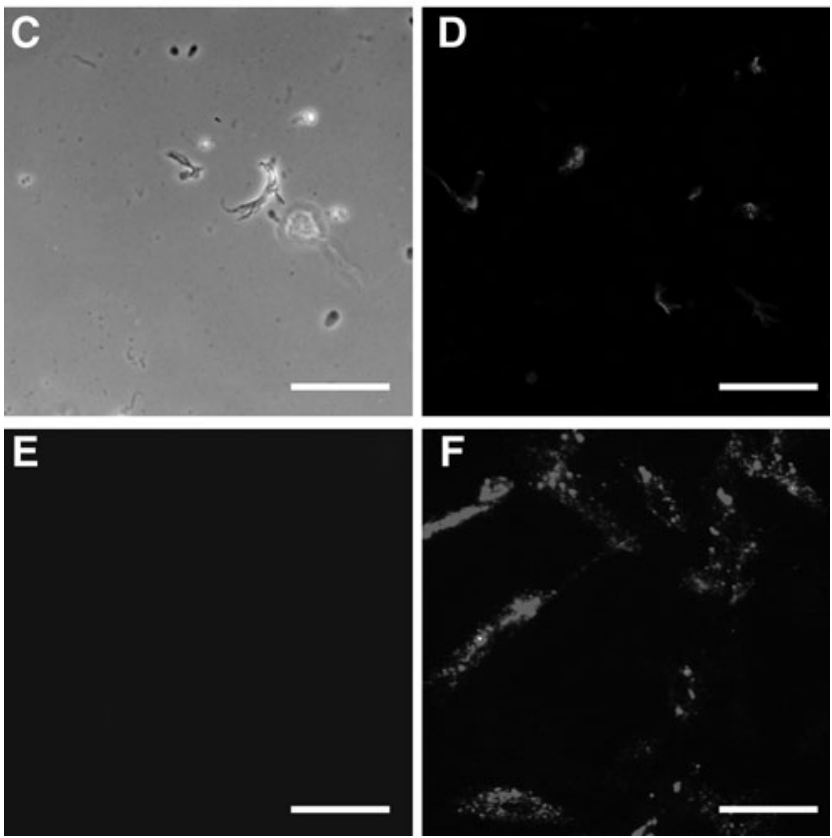

FIG. 3. The processes of the ADSCs labeling (A, B), lysis by hypotonicity and trypsin (C, D), and ADSCs debris coculturing with unlabeled (E)/labeled (F) ADSCs for 7 days under light microscopy and fluorescent microscopy. Bar $=100 \mu \mathrm{m}$.

unlabeled ADSCs emit red fluorescence (Fig. 3F) after being cocultured for 7 days in vitro, but also lead the livers, kidneys, spleens, brains, and peripheral blood to display red fluorescence under the fluorescent microscope when the PKH26-labeled cells debris was injected via the tail vein for 7 days (Fig. 4) in vivo.

Judging by the present use of PKH26, most of the researchers knew that the PKH26 was lipophilic, stable, and with a strong red fluorescent signal during tracing the transplanted cells in liver diseases [12], myocardial infarct model [13], and central nervous diseases [14], but the specificity of PKH26 to the transplanted cells was ignored. We knew that not all the transplanted cells in vivo can survive after administration because of the pathological microenvironment; therefore, the cells debris from PKH26-labeled transplanted cells was able to transfer the dye to the host cells and finally mistook the host cells (myocardial cells, neurons, or astrocytes) markers as the differentiation of the transplanted cells. Our results confirmed that the dead labeled cells debris could transfer their red fluorescence to the liver, spleen, kidney, brain, and peripheral blood with circulation by tail vein injection. The strong red fluorescence in the liver and kidney probably indicate that the dye metab-
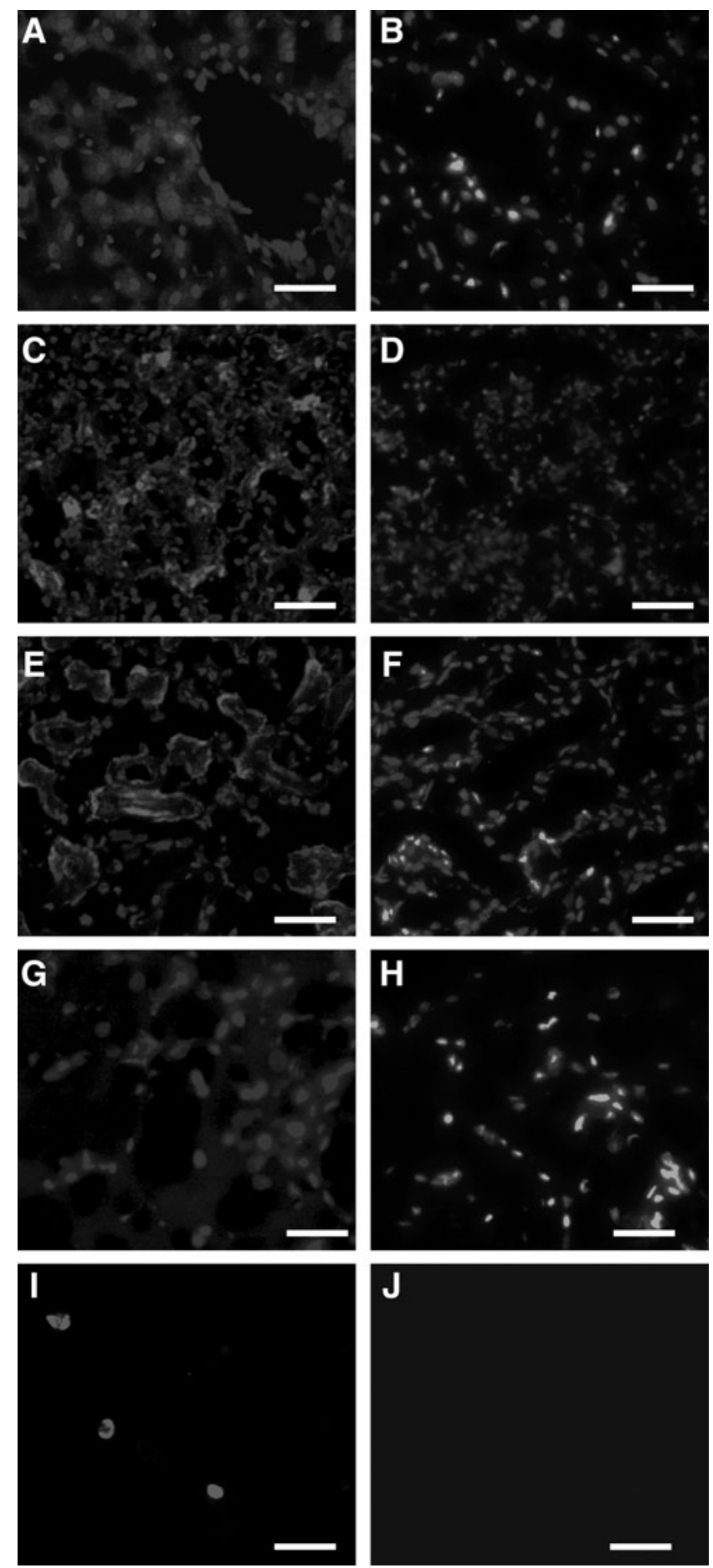

FIG. 4. The observation of red fluorescence in livers (A), spleens (C), kidneys (E), brains (G), and peripheral blood (I) after transplantation of cell debris labeling PKH26 for 7 days. The unlabeled cell debris was as a control, respectively $(\mathbf{B}, \mathbf{D}$, F, H, J). Bar $=100 \mu \mathrm{m}$.

olized or swallowed in these organs. Because of the brainblood barrier, the red fluorescence was weaker in brain tissue compared with other tissues, but it is enough to prove that the PKH26 can transfer the red dye to the host cells. Furthermore, during the experiment in vitro, the result that labeled cells debris could also deliver the dye to the unlabeled 
cells after co-cultured for 7 days kept consistent with the results in vivo.

So, in many transplanted studies using PKH26, for instance, $H$. Zhang reported that a number of cells carried the PKH26 membrane fluorescence in livers containing both hepatocytes and mature bile ducts after transplantation of embryonic small hepatocytes [15]. Gang Lu in 2009 also indicated by observing of red fluorescence that transplanted stem cells in myocardial infarct model survived on 7 days after PKH26-labeled cells engraftment [16]. Similarly, in the therapy study of central nervous system diseases, S.S. Seyed Jafari reported that PKH26-labeled stem cells could also be traced in brain and differentiated into cells expressing betatubulin III and S100 [6]. All these results above could be further confirmed according to our result and as early as in 2006, Thomas M. Coyne also indicated that 5-Bromo-2-deoxyUridine (BrdU) and bis benzamide (BBZ) could transfer donor label to host neurons and glia [17]. Therefore, as same as, PKH26 was localized to the host livers, spleens, kidneys, brains, and peripheral blood in the same way as BrdU and BBZ was. Use of the PKH26 also may provide a misleading index of donor survival and differentiation after transplantation.

Because of the inconsistency with respect to the mechanism of stem cells in vivo transplantation and the nonspecificity for cell tracer, we should draw more attention against the continuing use of PKH26 serials as cell tracer.

\section{Acknowledgments}

This work was supported by grants from the funds for National Key Clinic Department, the Natural Science Fund of China (No. 81171179; 81272439), the Funds for Key Sci-Tech Research Projects of Guangdong (No. 2008A030201019) and Guangzhou (No. 09B52120112-2009J1-C418-2, No. 2008A1E4011-6) to Professor Xiaodan Jiang.

\section{Author Disclosure Statement}

No competing financial interests exist.

\section{References}

1. Slezak SE and PK Horan. (1989). Fluorescent in vivo tracking of hematopoietic cells. Part I. Technical considerations. Blood 74:2172-2177.

2. Zeine R and T Owens. (1992). Direct demonstration of the infiltration of murine central nervous system by Pgp-1/ CD44high CD45RB(low) CD4 $+\mathrm{T}$ cells that induce experimental allergic encephalomyelitis. J Neuroimmunol 40:57-69.

3. Lansdorp PM. (1993). In vitro properties of purified human stem cell candidates. J Hematother 2:329-332.

4. Boutonnat J, M Barbier, K Muirhead, M Mousseau, D Grunwald, X Ronot and D Seigneurin. (2000). Response of chemosensitive and chemoresistant leukemic cell lines to drug therapy: simultaneous assessment of proliferation, apoptosis, and necrosis. Cytometry 42:50-60.

5. Zheng SX, YL Weng, CQ Zhou, ZZ Wen, H Huang, W Wu, JF Wang and T Wang. (2012). Comparison of cardiac stem cells and mesenchymal stem cells transplantation on the cardiac electrophysiology in rats with myocardial infarction. Stem Cell Rev [Epub ahead of print]; DOI: 10.1007/s12015012-9367-6.

6. Seyed JS, AA Ali, M Asadi-Shekaari, SN NematollahiMahani and V Sheibani. (2011). Investigating the effects of adult neural stem cell transplantation by lumbar puncture in transient cerebral ischemia. Neurosci Lett 495:1-5.

7. Kawaguchi K, Y Katsuyama, S Kikkawa, T Setsu and T Terashima. (2010). PKH26 is an excellent retrograde and anterograde fluorescent tracer characterized by a small injection site and strong fluorescence emission. Arch Histol Cytol 73:65-72.

8. Sun F, K Zhou, WJ Mi and JH Qiu. (2011). Combined use of decellularized allogeneic artery conduits with autologous transdifferentiated adipose-derived stem cells for facial nerve regeneration in rats. Biomaterials 32:8118-8128.

9. Tse KH, PJ Kingham, LN Novikov and M Wiberg. (2012). Adipose tissue and bone marrow-derived stem cells react similarly in an ischaemia-like microenvironment. J Tissue Eng Regen Med 6:473-485.

10. Chopp M and Y Li. (2002). Treatment of neural injury with marrow stromal cells. Lancet Neurol 1:92-100.

11. Taju G, SA Majeed, KS Nambi, BV Sarath, S Vimal, S Kamatchiammal and AS Hameed. (2012). Comparison of in vitro and in vivo acute toxicity assays in Etroplus suratensis (Bloch, 1790) and its three cell lines in relation to tannery effluent. Chemosphere 87:55-61.

12. Yan L, Y Han, J Wang, J Liu, Y He, H Wang and D Fan. (2008). Peripheral blood monocytes from the decompensated liver cirrhosis could migrate into nude mouse liver with human hepatocyte-markers expression. Biochem Biophys Res Commun 371:635-638.

13. Yoon J, BG Min, YH Kim, WJ Shim, YM Ro and DS Lim. (2005). Differentiation, engraftment and functional effects of pre-treated mesenchymal stem cells in a rat myocardial infarct model. Acta Cardiol 60:277-284.

14. Nakajima H, K Uchida, AR Guerrero, S Watanabe, D Sugita, N Takeura, A Yoshida, G Long, KT Wright, WE Johnson and H Baba. (2012). Transplantation of mesenchymal stem cells promotes an alternative pathway of macrophage activation and functional recovery after spinal cord injury. J Neurotrauma 29:1614-1625.

15. Zhang H, Z Liu, R Li, D Wang, W Liu, J Li, H Yu, F Zhang and K Dou. (2009). Transplantation of embryonic small hepatocytes induces regeneration of injured liver in adult rat. Transplant Proc 41:3887-3892.

16. Lu G, HK Haider, S Jiang and M Ashraf. (2009). Sca-1+ stem cell survival and engraftment in the infarcted heart: dual role for preconditioning-induced connexin-43. Circulation 119:2587-2596.

17. Coyne TM, AJ Marcus, D Woodbury and IB Black. (2006). Marrow stromal cells transplanted to the adult brain are rejected by an inflammatory response and transfer donor labels to host neurons and glia. Stem Cells 24:2483-2492.

Address correspondence to: Prof. Xiaodan Jiang Department of Neurosurgery Southern Medical University Zhujiang Hospital Guangzhou 510282

China

E-mail: jiangxiao_dan@163.com

Received for publication June 26, 2012

Accepted after revision August 22, 2012

Prepublished on Liebert Instant Online August 22, 2012 